\title{
Reverse Transcription Recombinase-aided Amplification Assay for H5 Subtype avian Influenza Virus
}

\section{Suchun Wang}

CAHEC: China Animal Health and Epidemiology Center

\section{Yang Li}

CAHEC: China Animal Health and Epidemiology Center

fuyou zhang ( $\nabla$ fyzhang0113@gmail.com )

China Animal Health and Epidemiology Center

\section{Nan Jiang}

Yanbian University Agriculture College

\section{Qingye Zhuang}

shandong vocational animal science and veterinary college

\section{Guangyu Hou}

CAHEC: China Animal Health and Epidemiology Center

\section{Lijian Jiang}

CAHEC: China Animal Health and Epidemiology Center

Jianmin Yu

CAHEC: China Animal Health and Epidemiology Center

\section{Xiaohui Yu}

CAHEC: China Animal Health and Epidemiology Center

\section{Hualei Liu}

CAHEC: China Animal Health and Epidemiology Center

\section{Chenglong Zhao}

CAHEC: China Animal Health and Epidemiology Center

\section{Liping Yuan}

CAHEC: China Animal Health and Epidemiology Center

\section{Baoxu Huang}

CAHEC: China Animal Health and Epidemiology Center

\section{Kaicheng Wang}

CAHEC: China Animal Health and Epidemiology Center https://orcid.org/0000-0003-0166-0951

\section{Research Article}


Keywords: avian influenza, H5 subtype, reverse transcription recombinase-aided amplification assay, single temperature, rapid, sensitive

Posted Date: December 28th, 2021

DOI: https://doi.org/10.21203/rs.3.rs-1147624/v1

License: (c) (i) This work is licensed under a Creative Commons Attribution 4.0 International License. Read Full License 


\section{Abstract}

Background: The H5 subtype avian influenza virus (AIV) has caused huge economic losses to the poultry industry and is a threat to human health. A rapid and simple test is needed to confirm infection in suspected cases during disease outbreaks.

Methods: In this study, we developed a reverse transcription recombinase-aided amplification (RT-RAA) assay for the detection of $\mathrm{H} 5$ subtype AIV. Assays were performed at a single temperature $\left(39^{\circ} \mathrm{C}\right)$, and the results were obtained within 20 min. Results: The assay showed no cross-detection with Newcastle disease virus or infectious bronchitis virus. The analytical sensitivity was $10^{3} \mathrm{RNA}$ copies/ $\mu \mathrm{L}$ at a $95 \%$ confidence interval according to probit regression analysis, with $100 \%$ specificity. Compared with published reverse transcription quantitative real-time polymerase chain reaction assays, the $\mathrm{k}$ value of the RT-RAA assay in 420 avian clinical samples was $0.983(p<0.001)$. The sensitivity for avian clinical sample detection was $97.26 \%(95 \% \mathrm{Cl}, 89.56-99.52 \%)$, and the specificity was $100 \%(95 \% \mathrm{Cl}, 98.64-100 \%)$.

Conclusions: These results indicated that our RT-RAA assay may be a valuable tool for detecting H5 subtype AIV.

\section{Introduction}

Influenza A virus (IAV), a member of the genus Influenza, family Orthomyxoviridae, contains eight singlestranded negative-sense RNA segments encoding at least 10 proteins [1, 2]. The IAV member avian influenza virus (AIV) can cause avian disease and public health problems. AIV can be classified into highly pathogenic avian influenza virus (HPAIV) and low pathogenic avian influenza virus (LPAIV) based on pathogenicity in chickens. The current HPAI H5N1 virus outbreak (from 2003 onwards) is, however, unprecedented in scale and geographic distribution, which have been prevalent among poultry in Asia, Europe and Africa. The viruses constantly undergo genetic drift and shift that permanently threatens the poultry industry and human health [3].

All LPAIV and HPAIV infections with H5 subtypes in poultry are notifiable to the World Organization for Animal Health [4]. Determination of the subtypes of AIV is of importance for the diagnosis of these infections, which can be achieved by analysis of the amino acid sequence of the cleavage site in the AIV hemagglutinin (HA) gene [5]. Besides, there are many methods, such as restriction enzyme cleavage patterns, probe hybridization and real time reverse transcription quantitative polymerase chain reaction (RTqPCR) $[5,6,7,8]$. Based on the widespread availability of RT-qPCR technology in diagnostic laboratories, it has been used to identify H5 subtype HPAIV of the goose/Guangdong (gs/GD) lineage.

Recently, rapid isothermal amplification techniques have been developed, such as loop-mediated isothermal amplification (LAMP) [7], recombinase polymerase amplification (RPA) [8], recombinase-aided amplification (RAA) and strand displacement amplification (SDA) [9], and widely used in clinical detection. Among these rapid nucleic acid detection methods, reverse transcription recombinase-aided amplification (RT-RAA) is a rapid thermostatic nucleic acid amplification technology that utilizes a recombinant enzyme obtained from bacteria or fungi. At room temperature, the recombinant enzyme can tightly bind to the primer DNA to form 
polymer. When the primer recognizes the template DNA for a complementary sequence that perfectly matches it, with the help of a single-stranded DNA binding protein, it opens the double-stranded structure of the template DNA under the action of DNA polymerase, a new cDNA strand is formed, and the amplification product grows exponentially [10]. This technology has the characteristics of high sensitivity, stronger specificity and reliability.

In this study, in order to efficiently detect H5 subtype AIV, an RT-RAA assay was designed and its analytical specificity and sensitivity were evaluated. Our study suggested that RT-RAA assay met the need of field testing, and presented a rapid and sensitive detection method that could be used as an alternative to animal inoculation or nucleotide sequencing.

\section{Materials And Methods \\ Ethics statement}

This study was conducted according to the animal welfare guidelines of the World Organization for Animal Health and was approved by the Animal Welfare Committee of the China Animal Health and Epidemiology Center (CAHEC). CAHEC has permission to study the activities of HPAIV. The swab samples were collected after being granted permission by several relevant parties, including the Ministry of Agriculture and Rural Affairs of China, CAHEC, the relevant veterinary sections of the provincial and national governments, and the relevant farm owners.

\section{Samples and extraction of viral nucleic acids}

All the nucleic acid of AIVs used in this study were identified in our laboratory (the National Avian Influenza Professional Laboratory in China Animal Health and Epidemiology Center) and were stored at $-80^{\circ} \mathrm{C}$. Newcastle disease virus (NDV) and infectious bronchitis virus (IBV) were maintained in our laboratory. The samples were centrifuged at $12,000 \times g$ for $10 \mathrm{~min}$ and the supernatant from each sample was used for RNA extraction on the QIAxtractor platform using a cador Pathogen 96 QIAcube HT kit (Qiagen). The extracted RNA was stored at $-80^{\circ} \mathrm{C}$ for subsequent tests.

\section{Preparation of viral RNA standard}

An $\mathrm{H} 5$ subtype AIV viral RNA standard was developed using the reference strain A/duck/Yunnan/5310/2006(H5N1) (GenBank accession number CY030889). A 1776-bp fragment of the whole HA gene of H5 subtype AIV was constructed and digested with enzyme. The digested plasmid was purified with DNA recovery kit (Thermo Fisher Scientific) and transcribed in vitro with RNA Production System-T7 kit. The products were extracted and purified by Rneasy MiNi Kit to remove the heteroproteins and various ions in the system. The concentration of cRNA standard transcribed in vitro was accurately measured by Nano Drop nucleic acid quantitative analyzer, and the copy number was calculated. The cRNA standard was continuously diluted with $\mathrm{RNase}$ free $\mathrm{H}_{2} \mathrm{O}$ in 10-fold gradient. The sensitivity of the RT-RAA 
was evaluated by real-time fluorescence detection, using the diluted cRNA standard ranging from $10^{6}$ $10^{1}$ copies/ $\mu \mathrm{L}$.

\section{Design of H5 RT-RAA primers and exo-probes}

To detect H5 subtype AIV, a total of 4636 available HA gene sequences of H5 subtype AIV obtained from GenBank database were aligned, which contained the HA gene sequence of all currently circulating branches of $\mathrm{H} 5$ subtype AIV, and highly conserved regions were subsequently identified with Molecular Evolutionary Genetics Analysis (MEGA) software 6.0 [11] for the design the gene-specific primers and probes. Primers were designed using OLIGO 7 software [12] and showed no major nonspecific sequence similarities by BLAST analysis. Three $\mathrm{H} 5$ forward primers and five reverse primers were designed to select the best primers and probes in combination. The appropriate primers and probe combination were selected by sequence analysis, which were shown in Table 1 . The 30th base at the $5^{\prime}$ end of the probe was labeled with the FAM fluorophore. The 30th base was connected to the abasic site tetrahydrofuran (THF). The 31st base was labeled with the BHQ1 quencher, and the 3 ' end was modified by $3 \otimes$ block. All the primers and probes were synthesized by Sangon Biotech.

Table 1

Primer and probe sequences used for RT-RAA and RT-qPCR assays

\begin{tabular}{|lllll|}
\hline Primer & Squence $\left(5^{\prime}-3^{\prime}\right)$ & $\begin{array}{c}\text { Size } \\
(\mathrm{bp})\end{array}$ & Gene & Source \\
H5- $\mathrm{F}$ & CAGTTTGAGGCYGTTGGAAGGGAATTTAAYAA & 32 & HA & $\begin{array}{l}\text { This } \\
\text { study }\end{array}$ \\
H5- R & CTTGTCRTAAAGGTTCTTGACATTTGAGTCAT & 32 & HA & $\begin{array}{l}\text { This } \\
\text { study }\end{array}$ \\
H5- P & $\begin{array}{l}\text { CTAGATGTCTGGACTTATAATGCTGAACT/i6FAMdT//THF/ } \\
\text { /iBHQ1dT/GGTTCTCATGGAAAAT[C3-spacer] }\end{array}$ & 47 & HA & $\begin{array}{l}\text { This } \\
\text { study }\end{array}$ \\
H5+1456 & ACGTATGACTATCCACAATACTCAG & 25 & HA & {$[13]$} \\
\hline H5-1685 & AGACCAGCTACCATGATTGC & 20 & HA & {$[13]$} \\
\hline H5+1637 & FAM-TCAACAGTGGCGAGTTCCCTAGCA-TAMRA & 24 & HA & [13] \\
\hline
\end{tabular}

\section{RT-RAA for detection of H5 subtype AIV}

The appropriate primers and exo-probes were screened, and the RT-RAA reaction was performed with an RT exo kit in $50 \mu \mathrm{L}$ reaction mixture including the necessary enzymes and reagents for RT and DNA amplification in lyophilized pellets (Jiangsu Qitian Bio-Tech Co. Ltd.). The reaction mixture contained the following: $2 \mu \mathrm{L}$ RNA template, $25 \mu \mathrm{L}$ rehydration buffer, $15.7 \mu \mathrm{L}$ deionized distilled water, $2.5 \mu \mathrm{L}$ magnesium 
acetate, $2.1 \mu \mathrm{L}$ each primer $(10 \mu \mathrm{M})$ and $0.6 \mu \mathrm{L}$ target-specific RT-RAA exo-probe (the probe concentration is $40 \mathrm{ng} / \mu \mathrm{L}$ ). For amplification, the tubes were then transferred to a tube holder in an RT-RAA fluorescence detection device (QT-RAA-F7200; Jiangsu Qitian Bio-Tech Co. Ltd.) set at $39^{\circ} \mathrm{C}$ for $20 \mathrm{~min}$. Each run included nuclease-free water as a negative control. The results of the RT-RAA interpretation criteria were determined by the slope of amplification curve of the RT-RAA fluorescence detection device (QT-RAA-F7200), the test result is positive when the slope $k$ value was greater than or equal to 20 , in addition, the test result is negative.

\section{Analytical sensitivity and analytical specificity of RT-RAA}

A dilution range of $\mathrm{H} 5$ subtype HA plasmid standard was used to select the appropriate RAA primers and exo-probe combination. The selected appropriate primers and exo-probes were verified by H5 RT-RAA analysis. To determine the analytical sensitivity of the H5 RT-RAA assay, we detected the H5 subtype AIV molecular RNA standard over a dilution range of $10^{7}-10^{1}$ copies/ $\mu \mathrm{L}$ under the optimal RT-RAA conditions, with eight replicates for each dilution.

The analytical specificity assay of the RT-RAA for $\mathrm{H} 5$ subtype AIV was evaluated using four H5-positive AIVs (one strain in clade 2.3.2.1, one in clade 7, and 55 in clade 2.3.4.4), 10 other subtype AIVs (H1N2, H3N2, H4N2, H6N2, H7N3, H7N9, H9N2, H10N7, H11N9), two NDVs and two IBVs. These viruses are the main respiratory viruses affecting birds and were previously identified by our laboratory. The details of all the viruses tested are listed in Table 2.

Table 2

Some information about the samples used for analytical sensitivity and analytical specificity assay in the study 


\begin{tabular}{|lllll|}
\hline Sample & Virus & HA subtype(clade) & H5 RT-RAA assay & H5 RT-qPCR assay \\
\hline K144 & AIV & H5N1(2.3.2.1) & + & + \\
\hline QD1 & AIV & H5N2(7) & + & + \\
G2324 & AIV & H5N6(2.3.4.4) & + & + \\
G2084 & AIV & H5N6(2.3.4.4) & + & + \\
\hline Q221 & AIV & H1N2 & - & - \\
\hline X1330 & AIV & H3N2 & - & - \\
\hline P174 & AIV & H4N2 & - & - \\
\hline A1267 & AIV & H6N2 & - & - \\
\hline H7N3 & AIV & H7N3 & - & - \\
\hline 1605 & AIV & H7N9 & - & - \\
\hline X169 & AIV & H9N2 & - & - \\
\hline H9 & AIV & H9N2 & - & - \\
\hline T55 & AIV & H10N2 & - & - \\
\hline S82 & AIV & H11N2 & - & - \\
\hline ND & NDV & / & - & - \\
\hline JS1816 & NDV & / & - & - \\
\hline M41 & IBV & / & - & - \\
\hline H52 & IBV & / & & - \\
\hline
\end{tabular}

\section{Detection and evaluation of clinical samples by H5 RT-RAA}

We collected about 420 oropharyngeal and cloacal swab samples from 29 sites in live poultry markets in six provinces, which were immediately placed in $1 \mathrm{~mL}$ antibiotic-containing PBS as described above and then stored at $-80^{\circ} \mathrm{C}$ until total nucleic acids were extracted with the above viral RNA extraction kit [21]. We evaluated the performance of the RT-RAA assay in 420 avian clinical samples and compared it with the published RT-qPCR method for H5 subtype AIV. The primers and probe of the RT-qPCR assay are listed in Table 1 [13]. The RT-qPCR assay was conducted with the One Step PrimeScript RT-PCR Kit (Takara).

\section{Statistical analysis}

To determine the RT-RAA detection limit, a probit analysis was performed at a 95\% confidence interval (CI), and the $k$ and $p$ values of RT-qPCR and RT-RAA were calculated [14]. In addition, we calculated the 
sensitivity and specificity of RT-qPCR and RT-RAA for detection in clinical samples of poultry. All statistical analyses were performed in SPSS 21.0 (IBM).

\section{Results}

\section{Analytical sensitivity of RT-RAA}

The sequences of the appropriate H5 RT-RAA primers and exo-probe are listed in Table 1. The detection results of RT-RAA sensitivity assay are shown in Figure 1. The primer and probe combinations designed by the present invention have a cRNA concentration of $10^{6}$ copies $/ \mu \mathrm{L}, 10^{5}$ copies $/ \mu \mathrm{L}, 10^{4}$ copies $/ \mu \mathrm{L}$, and $10^{3}$ copies $/ \mu \mathrm{L}, 10^{2}$ copies $/ \mu \mathrm{L}, 10^{1}$ copies $/ \mu \mathrm{L}$, a fluorescence amplification curve appears. Therefore, the detection limit of H5 RT-RAA assay was $10^{2}$ cRNA copies/ $\mu \mathrm{L}$ (Table 3 and Fig 1).

Table 3

Assay data used for probit analysis to calculate the detection limits of H5 RT-RAA

\begin{tabular}{|l|l|}
\hline copies/ $\mu \mathrm{L}$ & $\begin{array}{l}\text { No. of positive samples/ No. of samples tested by } \\
\text { the RT-RAA assays for detection of H5 subtype AIV }\end{array}$ \\
\hline $10^{6}$ & $\mathbf{8 / 8}$ \\
\hline $10^{5}$ & $\mathbf{8 / 8}$ \\
\hline $10^{4}$ & $\mathbf{8 / 8}$ \\
\hline $10^{3}$ & $\mathbf{5 / 8}$ \\
\hline $10^{2}$ & $\mathbf{2 / 8}$ \\
\hline $10^{1}$ & $\mathbf{0 / 8}$ \\
\hline a Each dilution was tested in a total of eight replicates.
\end{tabular}

\section{Analytical specificity of RT-RAA}

The test group corresponding to the H5 subtype AIV RNA template showed standard fluorescence detection curves, and the other virus test groups and negative control groups did not show amplification curves. Thus, the RT-RAA assay did not crossreact with other subtype AIVs, NDVs and IBVs and demonstrated high specificity for the detection of H5 subtype AIV (Fig 2). 


\section{1: K144 (H5N1); 2: QD1 (7); 3: G2324 (H5N6); 4: G2084 \\ (H5N6)}

\section{Evaluation of the RT-RAA for clinical samples}

The 420 conserved avian clinical samples collected from live poultry markets were tested by RT-RAA assay and compared with RT-qPCR. A threshold cycle value of 36 was used as the cut-off for a positive result in RT-qPCR. RT-qPCR detected 73 of the 420 samples as positive (17.38\%), while RT-RAA correctly identified and differentiated 71 positive samples, with a sensitivity of $97.26 \%$ (95\% Cl, 89.56-99.52\%) and 100\% specificity (95\% Cl, 98.64-100\%) (Table 3). The $\mathrm{k}$ value for RT-RAA and RT-qPCR was $0.983(p<0.001)$.

Table 3

Detection of H5 subtype AIV in avian clinical samples

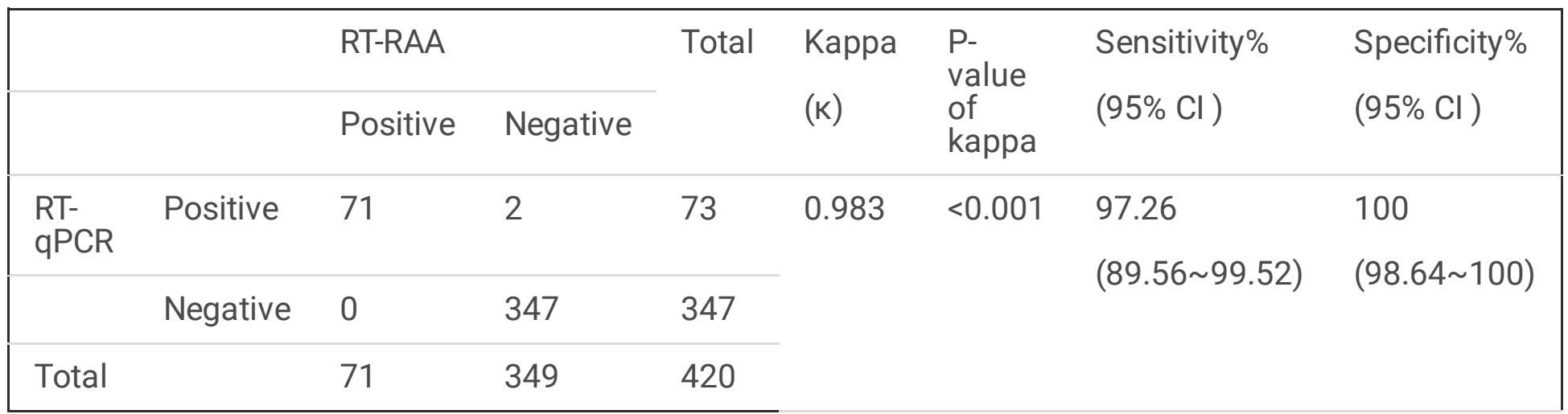

\section{Discussion}

The frequent variation of AIV antigen increases the difficulty of AIV detection. Among all the AIV subtypes, H5 subtype HPAIV often leads to high morbidity and mortality in poultry. Nowadays, the H5 subtype AIV is widely prevalent and of significant concern to the poultry industry and public health in China. Therefore, early detection of AIV and H5 subtype is necessary in the surveillance and control of AIV outbreaks. Until now, there have been many methods used in AIV detection, such as gold immunochromatographic assay [15], microarray [16], immunosensor [17], immunofluorescence [18] and enzyme-linked immunosorbent assay [19]. However, many methods require complex and costly devices and are difficult to perform in the field. So far, many detection methods have been used for rapid detection of the AIV in the field, such as nucleic acid sequence amplification [20], RT-LAMP and RT-RPA [22]. Moreover, as the mutation rate of the H5 subtype AIV accelerates, the previous detection methods may not meet the actual detection needs. Some of them fail to subtype AIV, while others cannot be applied in early diagnosis owing to inadequate 
sensitivity [23], so a rapid diagnostic method capable of detecting all avian influenza epidemic strains is needed.

RAA is a novel isothermal amplification and detection assay requiring only 20 min to complete, while RTqPCR and conventional PCR need longer time. In PCR, thermocycling is required for double-stranded DNA separation, primer binding and amplification. RT-RAA could produce a positive signal in as little as $4 \mathrm{~min}$ and be completed in $30 \mathrm{~min}$, at half the cost of RT-qPCR. RAA can be carried out using a portable device with no complicated processes, while the instruments for RT-qPCR and conventional PCR are more expensive. RAA can also use reverse transcriptase and a fluorescent probe system to detect RNA amplicons in real time [16]. Until now, RAA has widely been used for detecting human pathogens, including Salmonella, respiratory syncytial virus, coxsackievirus, hepatitis B virus and Schistosoma japonicum-specific gene fragments and there have been no previous reports of the use of RAA for detecting H5 subtype AIV [24, 25, $26,27]$.

In this study, an H5 RT-RAA assay was created, which indicated the potential value of our method for early detection and rapid diagnosis of the H5 subtype AIV. In the detection of experimental and clinical samples, this method showed higher sensitivity along with high efficiency. RT-qPCR detected 73 of the 420 samples as positive (17.38\%), while RT-RAA correctly identified 71 positive samples, with a sensitivity of $97.26 \%$ (95\% Cl, 89.56-99.52\%) and 100\% specificity (95\% Cl, 98.64-100\%) (Table 3). The $\mathrm{k}$ value for RT-RAA and RT-qPCR was $0.983(p<0.001)$. Moreover, the clinical swab samples detected as positive in RT-RAA were also certified as positive by PCR detection and sequencing. According to the primer/exo-probe design method and the sample detection results, the RT-RAA method was considered to be suitable for detecting almost all $\mathrm{H} 5$ subtypes. To the best of our knowledge, this is the first RT-RAA method for the detection of multiple $\mathrm{H} 5$ clades in AIV.

In conclusion, the RT-RAA method established in our study quickly and accurately identified H5 subtype AIV, including the current epidemic strains, which meets the need for H5 subtype AIV testing. This RT-RAA is expected to detect emerging $\mathrm{H} 5$ subtype AIV rapidly and provide a powerful and valuable tool for the control of $\mathrm{H} 5$ subtype AIV.

\section{Declarations}

\section{Ethics approval and consent to participate}

Not applicable.

\section{Consent for publication}

Not applicable.

\section{Availability of data and materials}


All data generated or analyzed during this study are included in this published article [and its supplementary information files].

\section{Competing interests}

The authors declare that they have no competing interests.

\section{Funding}

This work was supported by the National Key Research and Development Program of China (2017YFC120050)

\section{Authors' contributions}

Conceptualization: SW, YL, FZ, KW, HL, BH.

Data Curation: SW, K W.

Formal Analysis: SW, YL, FZ.

Funding Acquisition: KW, BH.

Investigation: SW, YL, FZ, NJ, QZ, GH.

Methodology: SW, YL, FZ, KW.

Project Administration: KW.

Resources: GH, QZ, LJ, JY, CZ, XY, LY.

Supervision: KW.

validation: SW, YL, FZ, NJ.

Visualization: SW, YL, FZ.

Writing-Original Draft Preparation: SW, YL, FZ.

Writing-Review \& Editing: SW, YL, FZ, K W, HL, BH.

\section{Acknowledgement}

Not applicable. 


\section{References}

1. Dadonaite B, Gilbertson B, Knight ML, Trifkovic S, Rockman S, Laederach A, Brown LE, Fodor E, Bauer DL. The structure of the influenza A virus genome. Nature Microbiology. 2019;4(11), 1781-1789.

2. Nakajima K. Influenza virus genome structure and encoded proteins. Nihon Rinsho. 1997;55, 25422546.

3. Peiris JSM, de Jong MD, Guan Y. Avian influenza virus (H5N1): a threat to human health. Clin. Microbiol. Rev. 2007;20, 243-267.

4. Drew TW. OIE manual of diagnostic test and vaccine for terrestrial animal. Paris, France: Avian Influenza. 2008;465-481.

5. Capua I, Alenxander DJ. Avian influenza and Newcastle disease: A filed and laboratory manual. Milan Italy: Springer Verlag Italia. 2009.

6. Sidoti F, Rizzo F, Costa C, Astegiano S, Curtoni A, Mandola ML, Rossana C, Massimiliano B. Development of real time RT-PCR assays for detection of type A influenza virus and for subtyping of Avian H5 and H7 hemagglutinin subtypes[J]. Molecular Biotechnology. 2010;44(1):41.

7. Notomi T, Okayama H, Masubuchi H, Yonekawa T, Watanabe K, Amino N, et al. Loop-mediated isothermal amplification of DNA. Nucleic Acids Research. 2000;28(12): E63.

8. Lutz S, Weber P, Focke M, Faltin B, Hoffmann J, Muller C, Mark D, Roth G, Munday P, Armes N, Piepenburg $\mathrm{O}$, Zengerle R, Stetten FV. Microfluidic lab-on-a-foil for nucleic acid analysis based on isothermal recombinase polymerase amplification (RPA). Lab on a Chip. 2010;10, 887-893.

9. Walker GT, Fraiser MS, Schram JL, Little MC, Nadeau JG, Malinowski DP. Strand displacement amplification-an isothermal,in vitro DNA amplification technique. Nucleic Acids Research. 1992;20, 1691-1696.

10. Bei L, Cheng HR, Yan QF, Huang ZJ, Shen GF, Zhang ZF, Li YN, Deng ZX, Lin M, Cheng Q. Recombinaseaid amplification, a novel technology of in vitro rapid nucleic acid amplification[J]. Scientia Sinica. 2010;40: 983-988.

11. Tamura K, Stecher G, Peterson D, Filipski A, Kumar S. MEGA6: Molecular evolutionary genetics analysis version 6.0. Molecular Biology and Evolution. 2013;30, 2725-2729.

12. Rychlik W. OLIGO 7 primer analysis software. Methods in Molecular Biology, 2007;402, 35-60.

13. Spackman E, Senne DA, Myers TJ, Bulage LL, Suarez DL. Development of a real-time Reverse reverse Transcriptase transcriptase PCR Assay assay for Type type A Influenza influenza virus and the avian H5 and H7 hemagglutinin subtypes[J]. Journal of Clinical Microbiology. 2002;40(9), 3256-3260.

14. Chen C, Li XN, Li GX, Zhao L, Duan SX, Yan TF, Feng ZS, Ma XJ. Use of a rapid reverse-transcription recombinase aided amplification assay for respiratory syncytial virus detection. Diagn Microbiol Infect Dis. 2018;90, 90-95.

15. Peng D, Hu S, Hua Y, Xiao Y, Li Z, Wang X, Bi DR. Comparison of a new gold-immunochromatographic assay for the detection of antibodies against avian influenza virus with hemagglutination inhibition and agar gel immunodiffusion assays. Veterinary Immunology \& Immunopathology. 2007;117(1-2), 17-25. 
16. Gall A, Hoffmann B, Harder T, Grund C, Höper D, Beer M. Design and validation of a microarray for detection, hemagglutinin subtyping, and pathotyping of avian influenza viruses. Journal of Clinical Microbiology. 2009;47(2), 327-34.

17. Wang R, Wang Y, Lassiter K, Li Y, Hargis B, Tung S, Berghman L, Bottje W. Interdigitated array microelectrode based impedance immunosensor for detection of avian influenza virus H5N1. Talanta. 2009;79(2), 159-64.

18. Zhao GY, Shu-Chun WU, Wang LN, Chen GB, Cui SJ. A disposable amperometric enzyme immunosensor for rapid detection of avian influenza virus. Chinese Journal of Animal \& Veterinary Sciences. 2008;39(10), 1442-8.

19. Song JL, Zhang WD, Wang JP, Zuo-Sheng LI, Feng ZL, Yuan-Yuan HU, Guo SH, Zhang YG, Fan QS, Song $X L$. Development of type and subtype-specific immunofluorescence techniques for detection of avian influenza virus. Journal of Yunnan University. 2008;30(5), 526-30.

20. Collins RA, Ko LS, Fung KY, Chan KY, Xing J, Lau LT. Rapid and sensitive detection of avian influenza virus subtype H7 using NASBA. Biochemical and Biophysical Research Communications. 2003;300, 507-515.

21. Cui D, Zhao D, Xie G, Yang X, Huo Z, Zheng S, Yu F, Chen Y. Simultaneous detection of influenza A subtypes of H3N2 virus, pandemic (H1N1) 2009 virus and reassortant avian H7N9 virus in humans by multiplex one-step real-time RT-PCR assay. SpringerPlus. 2016;5,2054.

22. Wahed AEA, Weidmann M, Hufert FT. Diagnostics-in-a-Suitcase: Development of a portable and rapid assay for the detection of the emerging avian influenza A (H7N9) virus. Journal of Clinical Virology. $2015 ; 69,16-21$.

23. Zhou EM, Cantin MF. Evaluation of a competitive ELISA for detection of antibodies against avian influenza virus nucleoprotein. Avian Diseases, 1998;42(3), 517-22.

24. Yan TF, Li XN, Wang L, Chen C, Duan SX, Qi JJ, Li LX, Ma XJ. Development of a reverse transcription recombinase-aided amplification assay for the detection of coxsackievirus A10 and coxsackievirus A6 RNA. Archives of Virology. 2018;163, 1455-1461.

25. Qi J, Li X, Zhang Y, Shen X, Song G, Pan J, Fan T, Wang RH, Li LX, Ma XJ. Development of a duplex reverse transcription recombinase-aided amplification assay for respiratory syncytial virus incorporating an internal control. Archives of Virology. 2019;164, 1843-1850.

26. Shen XX, Qiu FZ, She LP, Yan TF, Zhao MC, Qi JJ, Chen C, Zhao L, Wang L, Feng ZS, Ma XJ. A rapid and sensitive recombinase aided amplification assay to detect hepatitis $B$ virus without DNA extraction. BMC Infectious Diseases. 2019;19, 229.

27. Song Z, Ting L, Kun Y, Wei L, Jian-Feng Z, Li-Chuan G, Yan-Hong L, Yang D, Qing-Jie Y, Hai-Tao Y. Establishment of a recombinase-aided isothermal amplification technique to detect Schistosoma japonicum specific gene fragments. 2018;30, 273-277.

\section{Figures}


Figure 1

Analytical sensitivity of the H5 RT-RAA assay. A dilution range from $10^{6}$ to $10^{1} \mathrm{copies} / \mu \mathrm{L}$ of H5 subtype AIV cRNA was used to evaluate the detection limit of H5 RT-RAA assay. Negative represents negative control. 16: $10^{6}$ copies $/ \mu \mathrm{L}-10^{1}$ copies $/ \mu \mathrm{L} ; 7$ : negative control.

Figure 2

Analytical specificity of the H5 RT-RAA assay. Detection signals were recorded by real-time fluorescence RTRAA with four samples including H5 subtype AIVs (H5N1, H5N2 and H5N6), while no signals were detected from the 14 samples including other subtype AIVs, NDVs, IBVs and negative controls.

1: K144 (H5N1); 2: QD1 (7); 3: G2324 (H5N6); 4: G2084 (H5N6) 